jaundice to encephalopathy $<7$ days), 8 Subacute/Acute Liver Failure (S/ALF: jaundice to encephalopathy $>7$ days), and 18 healthy controls (HC). Flow cytometery was performed to investigate monocyte phenotype (CD14, CD16, HLA-DR, CD86, CD163, CD11b and CCR5)

Results Compared to HC, total monocyte count was elevated in S/ ALF, but reduced in hyperALF $(p<0.001)$, while CD14hi/CD16+ monocytes were expanded in percentage of total monocytes and absolute numbers in S/ALF $(17.4 \% ; 0.14)$ compared to HC (4\%; $0.014)(p<0.001)$. Although the percentage of CD14hi/CD16+ monocytes in hyperALF was higher $(5.6 \%, \mathrm{p}<0.01)$, the absolute number (0.013) was similar to HC. Though all CD14hi/CD16+ monocytes expressed HLA-DR, the Mean Fluorescence Intensity (MFI) was reduced compared to HC $(<0.001)$. HyperALF CD14hi/ CD16+ monocytes had lower HLA-DR MFI compared to S/ALF $(p<0.001)$. A similar pattern was seen for CD86 expression $(p<0.01)$. CD14hi/CD16+ monocytes showed increased expression of CD163 in hyperALF but not in S/ALF compared to HC $(<0.01)$. Compared to HC, CD11b and CCR5 were up-regulated in all ALF groups $(\mathrm{p}<0.001)$.

Conclusion We have demonstrated an expansion of CD14hi/CD16+ monocytes in ALI with an activated phenotype for adhesion and migration. CD14hi/CD16+ monocytes phenotypically resemble M1 macrophages in S\ALF, possibly reflecting a pathogenic role in the perpetuation of liver injury, while they resemble M2 in hyperALF and may be instrumental to the resolution of liver injury. This distinction requires further investigation should therapeutic strategies to target monocyte migration be attempted.

Competing interests None declared.

\section{PTU-016 ALBUMIN RESTORES RENAL BLO0D FLOW (RBF) AUTOREGULATION IN PATIENTS WITH REFRACTORY ASCITES AND ACUTE-ON-CHRONIC LIVER FAILURE (ACLF) THROUGH STABILISATION OF ENDOTHELIAL FUNCTION}

doi:10.1136/gutjnl-2012-302514c.16

R Garcia Martinez, ${ }^{*}$ J O'Brien, L Noiret, N Davies, R Mookerjee, R Jalan. Medicine, UCL Hepatology, London, UK

Introduction Haemodynamic alterations in liver failure are associated with endothelial dysfunction, a pro-inflammatory state and sympathetic activation which lead to disturbed RBF autoregulation and renal failure. Albumin is a multifunctional protein that has been shown in several studies to prevent and treat renal dysfunction in patients with advanced cirrhosis and liver failure. We hypothesised that the beneficial effects of albumin in cirrhosis is likely to be through mechanisms in addition to volume expansion. The aims of the study were to investigate the effects of albumin on systemic and renal haemodynamics, inflammation and endothelial dysfunction in refractory ascites and patients with acute kidney injury (AKI) in the setting of ACLF.

Methods Twenty-two patients were recruited [Group 1, $n=12$, refractory ascites; Group 2, $n=10$ patients with AKI admitted with an acute deterioration of their liver function due to either alcoholic hepatitis or infection]. Both groups were treated with Albumin $60 \mathrm{~g} / \mathrm{d}$ over 3-4 days. Cardiac output (CO) and renal blood flow (RBF) haemodynamics were measured. Endothelial dysfunction was assessed through measurement of von Willebrand factor (vWF) and serum nitrite (NO) levels. F2 $\alpha$ Isoprostanes (F2 $\alpha$ ), resting neutrophil burst and Interleukin (IL)- 6 were quantified as markers of oxidative stress, endotoxemia and inflammation respectively.

Results Albumin therapy was associated with significant improvements in haemodynamic parameters (increased RBF, MAP, decreased
$\mathrm{CO}, \mathrm{HR} ; \mathrm{p}<0.05)$ which resulted in a shift in the RBF autoregulation curve towards normalisation (Abstract PTU-016 figure 1). In parallel, improvement of renal dysfunction (creatinine, creatinine clearance and $\mathrm{Na}+$ excretion; $\mathrm{p}<0.05$ each), sympathetic activation (noradrenaline levels; $\mathrm{p}<0.01$ ), inflammation/oxidative stress (F2 $\alpha$ and neutrophil burst; $p<0.05$ ), endothelial dysfunction (vWF and $\mathrm{NO}$ metabolism $\mathrm{p}<0.05$ ) and the functional capacity of albumin (IMAR $p<0.005$ ) was observed. Restoration of RBF correlated inversely with change in $\mathrm{vWF}\left(\mathrm{r}^{2}=0.55, \mathrm{p}<0.001\right)$.

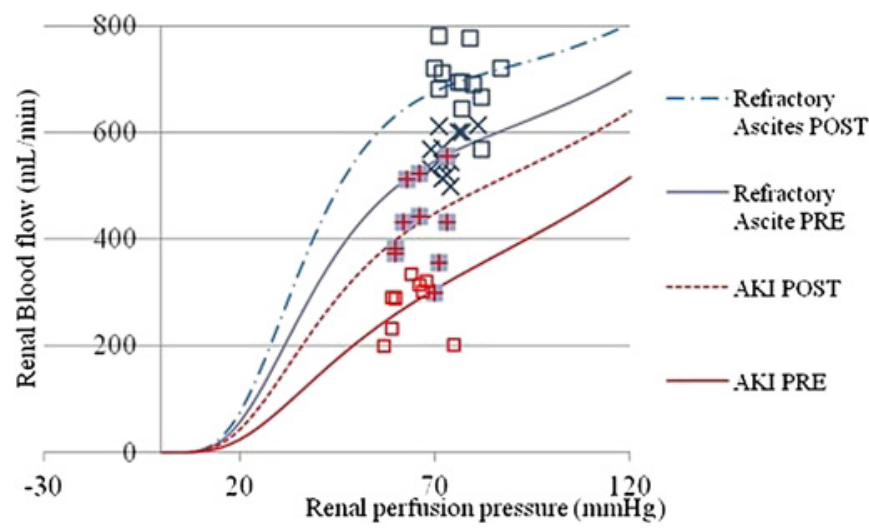

Abstract PTU-016 Figure 1

Conclusion This study suggests that albumin infusion improves albumin function which has pleiotropic effects and results in a reduction in inflammation and improvement in endothelial function leading to improved systemic haemodynamics and renal blood flow autoregulation.

Competing interests None declared.

\section{PTU-016a FUNCTIONAL DEFECTS IN CIRCULATING MONOCYTES MAY CONTRIBUTE TO SUSCEPTIBILITY TO INFECTION IN ALCOHOLIC HEPATITIS}

doi:10.1136/gutjnl-2012-302514c.16a

${ }^{1} \mathrm{~N}$ Vergis, ${ }^{*} \mathrm{C} \mathrm{G}$ Antoniades, ${ }^{2} \mathrm{~V}$ Zingarelli, ${ }^{2} \mathrm{R} \mathrm{D}$ Abeles, ${ }^{2} \mathrm{Y} \mathrm{Ma},{ }^{2} \mathrm{D}$ Shawcross, ${ }^{2} \mathrm{~A}$ Janmohamed, ${ }^{3} \mathrm{D}$ Patch, ${ }^{3} \mathrm{~W}$ Rosenberg, ${ }^{4} \mathrm{P}$ Kennedy, ${ }^{4} \mathrm{G}$ Foster, ${ }^{1} \mathrm{~S}$ Khan, ${ }^{2} \mathrm{~K}$ Agarwal, ${ }^{2} \mathrm{M}$ Heneghan, ${ }^{2} \mathrm{~J}$ 0'Grady, ${ }^{2} \mathrm{D}$ Vergani, ${ }^{2} \mathrm{~J}$ Wendon, ${ }^{1} \mathrm{M}$ Thursz. ${ }^{1} \mathrm{Hepa}$ tology and Gastroenterology, Imperial College, London, UK; ${ }^{2}$ Institute of Liver Studies, King's College Hospital, London, UK; ${ }^{3}$ Hepatology, Royal Free Hospital, London, UK; ${ }^{4}$ Hepatology, Royal London Hospital, London, UK

Introduction Infection is common in patients with severe alcoholic hepatitis (AH) and a significant contributor to mortality. Monocytes play an important role in bacterial elimination by phagocytosis, using intracellular oxidative killing and antigen presentation. Our study sought to evaluate monocyte phagocytosis and scavenger receptor expression in $\mathrm{AH}$.

Methods Monocytes were collected from 14 patients with AH (DF $>31$, prior to treatment) and 22 healthy controls (HC). Using FACS, monoclonal antibodies to scavenger receptors (CD36, -64, -163, -206, DCIR) and HLA-DR were used for immunophenotyping. Subsequently, ex-vivo monocyte phagocytosis and oxidative burst activity was assessed using FITC-labelled opsonised and non-opsonised Escherichia coli.

Results The expression of scavenger receptors was deranged. In CD14+CD16- (classical) monocytes, CD163 MFI was reduced in $\mathrm{AH}$ compared to controls (587 vs 403; $\mathrm{p}=0.05$ ). CD36, -206 and DCIR expression was similar between $\mathrm{HC}$ and $\mathrm{AH}$ patients but CD64 MFI was raised (6002 vs 12599; $p<0.001$ ). The proportion of monocytes phagocytosing $E$ coli was lower in AH compared to HC 